\title{
Editorial
}

\section{Spin Transport and Magnetism in Low-Dimensional Materials}

\author{
Xiaohong Xu, ${ }^{1,2}$ Jingsheng Chen, ${ }^{3}$ Gillian A. Gehring, ${ }^{4}$ Xiangshui Miao, ${ }^{5}$ and Hao Zeng ${ }^{6}$ \\ ${ }^{1}$ Key Laboratory of Magnetic Molecules and Magnetic Information Materials of Ministry of Education and \\ School of Chemistry and Materials Science, Shanxi Normal University, Linfen 041004, China \\ ${ }^{2}$ Research Institute of Materials Science, Shanxi Normal University, Linfen 041004, China \\ ${ }^{3}$ Department of Materials Science and Engineering, National University of Singapore, Singapore 117576 \\ ${ }^{4}$ Department of Physics and Astronomy, University of Sheffield, Sheffield S3 7RH, UK \\ ${ }^{5}$ School of Optical and Electronic Information, Huazhong University of Science and Technology, Wuhan 430074, China \\ ${ }^{6}$ Department of Physics, University at Buffalo, State University of New York, Buffalo, NY 14260, USA \\ Correspondence should be addressed to Xiaohong Xu; xuxh@sxnu.edu.cn
}

Received 22 January 2017; Accepted 23 January 2017; Published 28 February 2017

Copyright (c) 2017 Xiaohong Xu et al. This is an open access article distributed under the Creative Commons Attribution License, which permits unrestricted use, distribution, and reproduction in any medium, provided the original work is properly cited.

Spin generation, manipulation, and detection in low-dimensional materials have entered a dynamic new phase. The continued miniaturization of magnetic storage and the integration of magnetic devices into current IC chip present new challenges for materials and device scientists, which require continued innovation in material structures as well as in device and system concepts for spin-dependent transport and magnetism. Recent advances in this field include the magnetoresistance effect, diluted magnetic semiconductors, Hall effect, field-free magnetization switching, improved hard magnetic materials, and the exploration of low-dimensional materials with controlled spin states.

The purpose of this special issue is to shed light on some of the current works being done on novel synthesis methods and the physical phenomena occurring in nanoscale magnetic materials that will be used in next-generation spintronic devices. Among the submissions, six papers were selected for publication in this special issue. The theoretical paper by H. B. Huang et al. reported the strain-assisted spin transfer torque (STT) induced magnetization switching in CoFeBbased magnetic tunnel junctions by combining phase-field method with micromagnetic simulations. This work indicates a new aspect of free field magnetization switching and compares it to the previously reported methods such as spin orbital torque combined with exchange bias and broken lateral inversion symmetry. G. Yang et al. reported the ultrasensitive anomalous Hall effect (AHE) in Ta/CoFe/oxide $\left(\mathrm{MgO}\right.$ and $\left.\mathrm{HfO}_{2}\right) / \mathrm{Ta}$ multilayers and discussed the effect of the annealing process on sensitivity. AHE sensitivity as high as $18792 \Omega / \mathrm{T}$ in the as-deposited $\mathrm{Ta} / \mathrm{CoFe} / \mathrm{MgO} / \mathrm{Ta}$ was obtained. This work gives a new insight that both the selection of the oxide material and the postannealing treatment play an important role in determining the sensitivity of AHE. The microstructure and magnetic properties of $\mathrm{NdFeB}$ films through $\mathrm{Nd}$ surface diffusion process were investigated by W. Liu et al., showing that the microstructure and magnetic properties of $\mathrm{Ta} / \mathrm{Nd} / \mathrm{NdFeB} / \mathrm{Nd} / \mathrm{Ta}$ films are strongly dependent on the NdFeB layer thickness.

The other three papers focused on the magnetoresistance effect of the multilayers and nanowire arrays. X. Liu et al. reported linear magnetoresistance in $\mathrm{Ll}_{0}-\mathrm{FePt} / \mathrm{ZnO} / \mathrm{Fe}$ multilayers, in which linear response is observed in a large range from $+5 \mathrm{kOe}$ to $-5 \mathrm{kOe}$. This type of linear magnetoresistance is significant for high field linear magnetic sensors. $\mathrm{L}$. $\mathrm{Xu}$ et al. reported the magnetoresistance properties of black phosphorus (BP) spin-valve devices consisting of thin $\mathrm{BP}$ flakes contacted by NiFe ferromagnetic electrodes. The devices show spin-valve effect from room temperature to low temperature with magnetoresistance of $0.57 \%$ at $4 \mathrm{~K}$. Highly ordered arrays of $\mathrm{Co} / \mathrm{Cu}$ multilayered nanowires were investigated by J. Han et al. using porous anodic alumina (PAA) templates. They discussed the effects of repeat period number and the thickness of the copper layer on the magnetic and magnetoresistance. The editors are sure that this group of papers will be a useful reference for future workers seeking further developments of magnetic nanodevices. 


\section{Acknowledgments}

We would like to thank all the authors and coauthors who submitted their papers to the special issue. We hope that the publications of this special issue will be of reference value for readers.

Xiaohong Xu Jingsheng Chen Gillian A. Gehring Xiangshui Miao Hao Zeng 

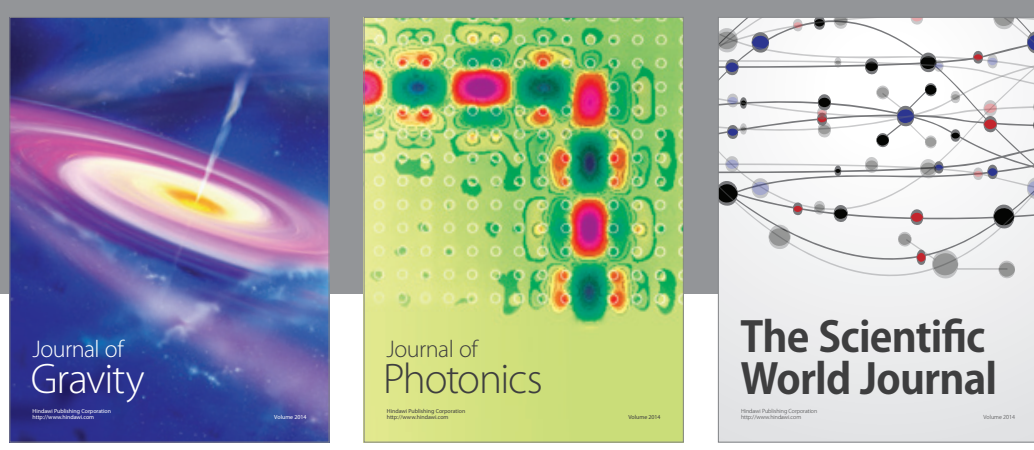

The Scientific World Journal
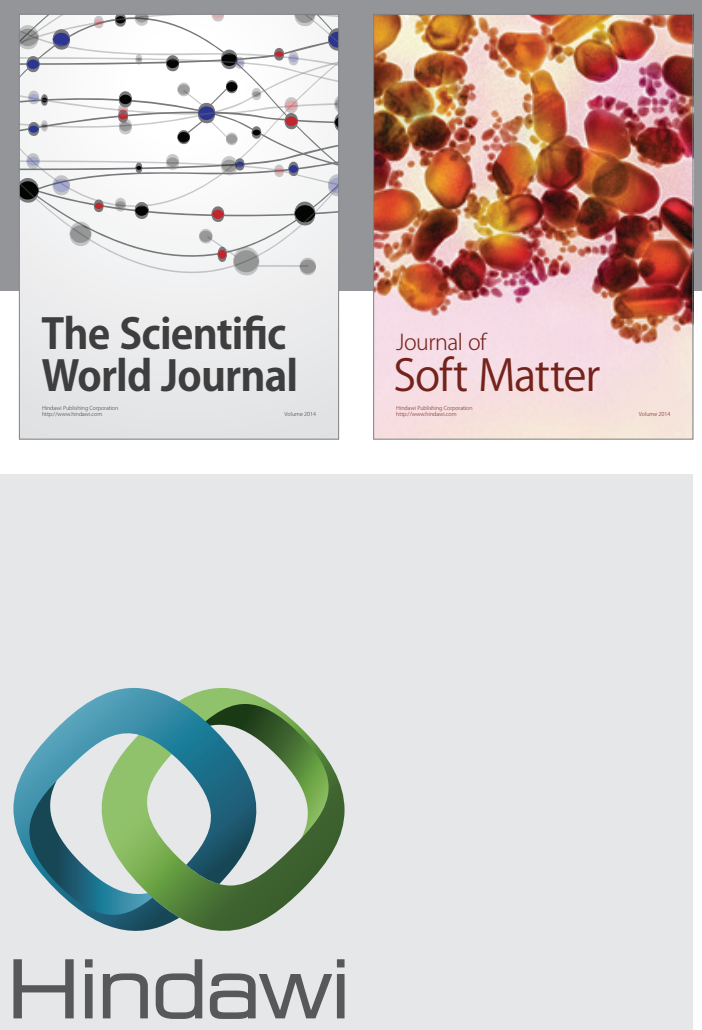

Submit your manuscripts at

https://www.hindawi.com
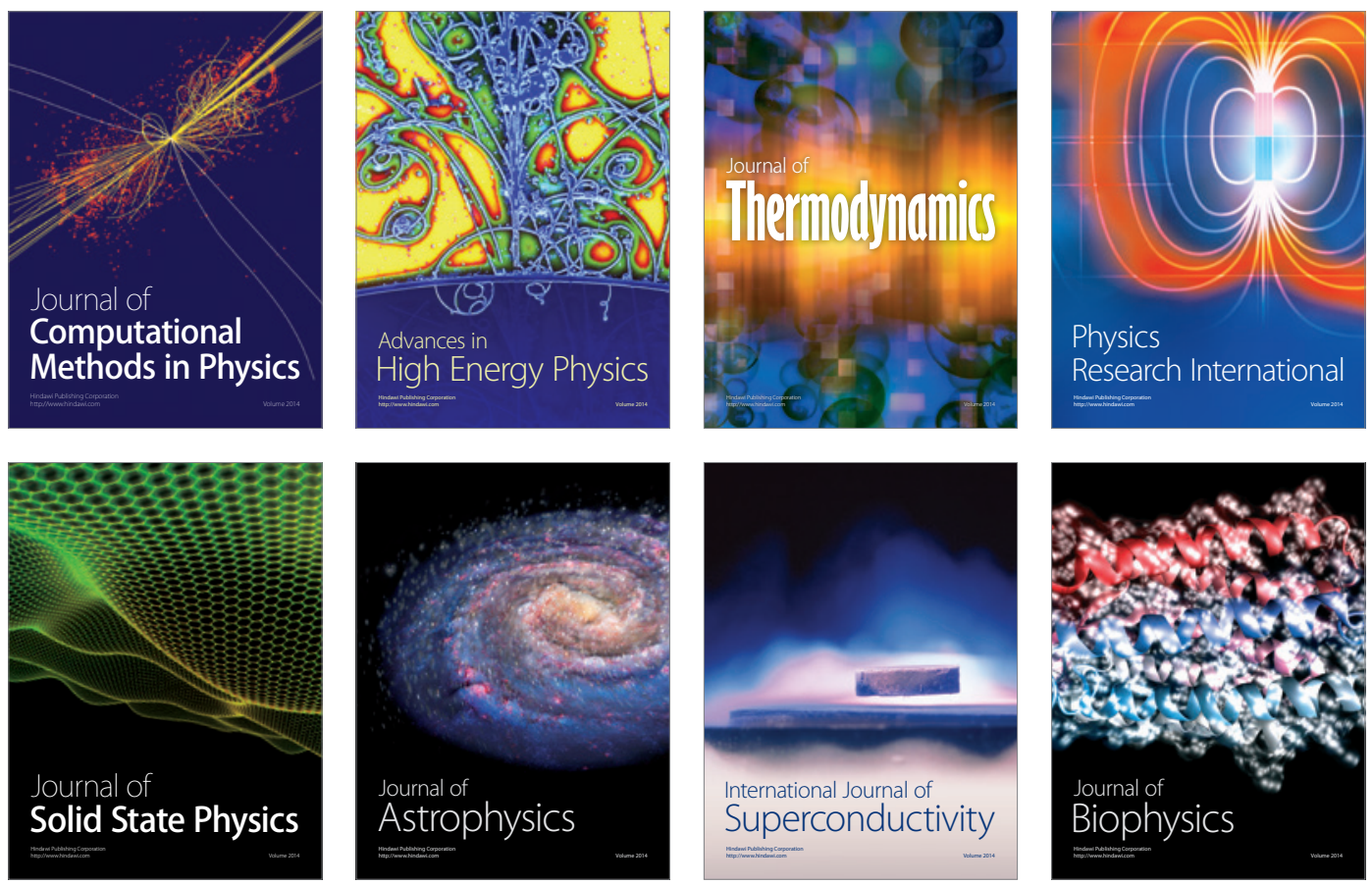
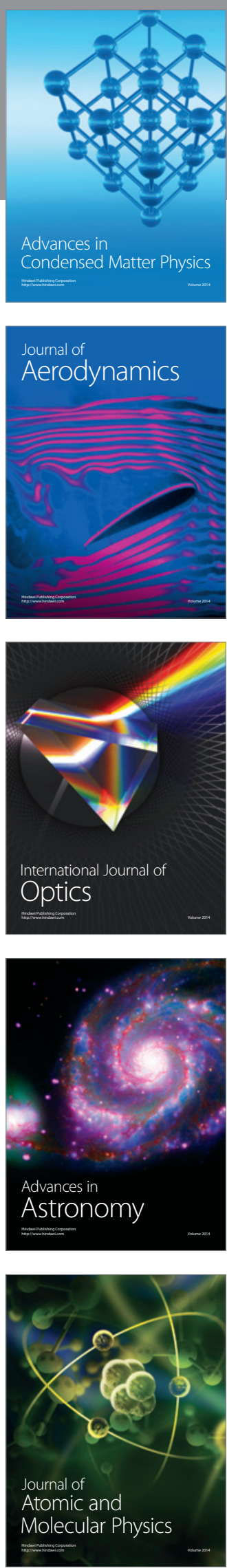